\title{
A case of mumps complicated by meningo-encephalomyelitis and appendicitis
}

\author{
M N Lucas ${ }^{1}$, S Dias $^{2}$ \\ Sri Lanka of Journal of Child Health, 2007; 36: 69-71
}

(Key words: mumps, meningo-encephalomyelitis, appendicitis)

\section{Introduction}

Mumps is known to cause complications such as meningo-encephalomyelitis, orchitis, epidydimitis, oophoritis, pancreatitis, thyroidtis, myocarditis, deafness, ocular complications and arthritis ${ }^{1,2}$. It is known that mumps, complicated by right sided orchitis and oophoritis, is commonly mistaken for appendicits ${ }^{1,2}$. However, it is less well known that mumps is listed as one of the risk factors as well as a causative virus for appendicitis ${ }^{3,4}$.

\section{Case report}

A 10 year old girl presented to ward 6B, General Hospital, Kalutara on $3^{\text {rd }}$ August, 2006, with headache, fever and vomiting of one day duration. She had a severe frontal headache associated with neck pain. There was no photophobia. She had vomited 11 times, the vomitus containing food particles but no blood. Five days back, she had developed a left sided parotid swelling suggestive of mumps. There was a strong contact history with many of her classmates, too, having mumps. She had not been immunized with MMR vaccine.

On admission, she was ill looking, febrile and had neck stiffness. Kernigs sign was negative and her vital signs were stable. She had a slight swelling over the left parotid area. A presumptive diagnosis of meningitis was made and treatment instituted. Her investigation results were as follows: C-reactive protein $<12 \mathrm{mg} / 1$, white cell count $10.8 \times 10^{9} / 1(\mathrm{~N}$ $78 \%$, L 20\%, E 2\%), random blood sugar $87 \mathrm{mg} / \mathrm{dl}$, CSF turbid, (protein $75 \mathrm{mg} / \mathrm{dl}, \mathrm{N}$ 65, L 625, RBC 75, sugar $44 \mathrm{mg} / \mathrm{dl}$, culture - no growth). Treatment was initiated with intravenous crystalline penicillin $2 \mathrm{~g} 6$ hourly and ceftriaxone $3.5 \mathrm{~g}$ daily on $4^{\text {th }}$ August.

She complained of a vague abdominal pain on $7^{\text {th }}$ August. There were no features of acute pancreatitis /

${ }^{1}$ Paediatric Registrar, ${ }^{2}$ Consultant Paediatrician, General Hospital, Kalutara.

(Received on 27 September, 2006. Accepted on 10 October 2006) oophoritis at the time. Ultrasound scan (USS) of the abdomen revealed no abnormality. However, her serum amylase was 1187 Somogyi units/l (normal range $<300$ ). On $8^{\text {th }}$ August, she developed periumbilical colicky pain which shifted to the right iliac fossa (RIF). It was not associated with nausea or vomiting. Her fever, which was coming down gradually, started to spike again the same day. Bowel habits were normal and she did not have any urinary symptoms. RIF tenderness, maximal at McBurney point, was found. At this stage, the possibility of acute appendicitis was considered. The USS of abdomen was repeated and an echogenic mass, resembling an inflamed appendix, was seen. She was referred to the general surgeon who made a clinical diagnosis of acute appendicitis warranting immediate surgery. Appendicectomy was carried out on $8^{\text {th }}$ August. A perforated, inflamed appendix, with minimal peritoneal contamination, was found. Intravenous metronidazole was added to the treatment regime and was given for 5 days. The antimeningitic treatment was continued for 14 days. She had an uneventful recovery and sat the year 5 scholarship examination 2 days before discharge. She was confirmed to have mumps IgM antibody by an enzyme immunoassay (EIA) test done at the Medical Research Institute.

\section{Discussion}

Mumps is a systemic illness caused by the paramyxovirus, transmitted by respiratory droplets. It has an incubation period of 14-25 days after which prodromal symptoms occur lasting for 3-5 days ${ }^{1}$. After the prodrome, symptoms of the virus depend on the organ affected. The most common presentation is a parotitis occuring in $30-40 \%$ of patients ${ }^{1}$. Other reported sites of infection are the testes, pancreas, eyes, ovaries, central nervous system, joints, and kidneys ${ }^{1}$. A patient is considered infectious from about 3 days before onset up to 4 days into active parotitis. Infections can be asymptomatic in up to $20 \%$ of persons ${ }^{1}$.

The clinical case-definition of mumps is an acute onset of unilateral or bilateral, tender, self-limited swelling of the parotid or other salivary gland lasting 
for more than 2 days without other apparent cause ${ }^{2}$. A lymphocytosis or leucopenia may be present ${ }^{1}$. Serum amylase level may be elevated ${ }^{1}$. CNS infections usually exhibit a lymphocytic pleocytosis ${ }^{1}$.

Serology is the simplest method for confirming mumps virus infection and EIA is the most commonly used test being more sensitive than other serologic tests ${ }^{2}$. It is available for both IgM and IgG. IgM antibodies usually become detectable during the first few days of illness and reach a peak about a week after onset. However, mumps IgM may be transient or missing in persons who have had any doses of mumps-containing vaccine ${ }^{2}$. Sera should be collected as soon as possible after symptom onset for IgM testing or as the acute-phase specimen for IgG seroconversion. Convalescent-phase sera should be collected 2 weeks later ${ }^{2}$.

Central nervous system (CNS) involvement in the form of meningo-encephalomyelitis is common, occurring asymptomatically (inflammatory cells in cerebrospinal fluid) in $50 \%-60 \%$ of patients ${ }^{2}$. Symptomatic meningo-encephalomyelitis (headache, stiff neck) occurs in up to $15 \%$ of patients and resolves without sequelae in 3-10 days ${ }^{2}$. Boys are more commonly affected than girls (3:1 ratio). Parotitis may be absent in as many as $50 \%$ of such patients $^{2}$.

Orchitis is the most common complication in postpubertal males. It occurs in as many as $50 \%$ of postpubertal males, usually after parotitis, but may precede it, begin simultaneously, or occur alone ${ }^{2}$. It is bilateral in approximately $30 \%$ of affected males. Approximately $50 \%$ of patients with orchitis have some degree of testicular atrophy, but sterility is rare $^{2}$. Oophoritis occurs in $5 \%$ of postpubertal females. It may mimic appendicitis. There is no relationship to impaired fertility ${ }^{2}$.

Pancreatitis is infrequent, but occasionally occurs without parotitis; the hyperglycemia is transient and is reversible ${ }^{2}$. Although single instances of diabetes mellitus have been reported, a causal relationship with mumps virus infection has yet to be conclusively demonstrated; many cases of temporal association have been described both in siblings and individuals, and outbreaks of diabetes have been reported a few months or years after outbreaks of mumps ${ }^{2}$.

Deafness caused by mumps virus occurs in approximately 1 per 20,000 reported cases. Hearing loss is unilateral in approximately $80 \%$ of cases and may be associated with vestibular reactions. Onset is usually sudden and results in permanent hearing impairment ${ }^{2}$. Electrocardiogram changes compatible with myocarditis are seen in 3-15\% of patients with mumps, but symptomatic involvement is rare. Complete recovery is the rule, but deaths have been reported $^{2}$. Other less common complications of mumps include arthralgia, arthritis, and nephritis ${ }^{2}$.

The relationship between mumps and appendicitis is little known. Mumps is stated both as a risk factor and as a virus in the aetiology of appendicitis ${ }^{3,4,5,6}$.

\section{Acknowledgement}

We thank Dr Mahanama Gunasekera, Consultant Surgeon, General Hospital, Kalutara for performing surgery on this patient.

\section{References}

1. Curtis KA. Mumps. Emedicine. (C) 1996-2006 by WebMD. Last Updated: July 12, 2006 Available from http://www.emedicine.com/EMERG/topic324.htm

2. Mumps virus: Pathogenesis and clinical features. Centres for disease control and prevention .USA: Department of health and human services. Last reviewed July 16, 2006. Available from http://www.cdc.gov/nip/publications/pink/mumps.rt f

3. Risk factors for acute appendicitis. CureResearch.com (C) 2000-2005 Adviware Pty Ltd. Last updated 9th April 2003. Available from: http://www.CureResearch.com/a/acute_appendicitis /riskfactors.htm

4. Jackson RH, Gardner PS, Kennedy J, McQuillin J. Viruses in the aetiology of acute appendicitis. Lancet. 1966; 2 (7466):711-4.

5. Associated conditions of mumps. WrongDiagnosis.com (C) 2000-2006 Adviware Pty Last updated: 21 September, 2006. Available from: http://www.wrongdiagnosis.com/m/mumps/assoc.h tm

6. Risk factors for acute appendicitis. WrongDiagnosis.com (C) 2000-2006 Adviware Pty. Last updated: 21 September, 2006. Available from: http://www.wrongdiagnosis.com/a/acute_appendici tis/riskfactors.htm 